\title{
Log Triviality in the Nambu - Jona-Lasinio Model
}

\author{
Simon Hands ${ }^{\mathrm{a} *}$ \\ ${ }^{\text {a}}$ Department of Physics, University of Wales Swansea, \\ Singleton Park, Swansea SA2 8PP, United Kingdom
}

Results are presented from a Monte Carlo simulation of the Nambu - Jona-Lasinio model with $\mathrm{SU}(2) \otimes \mathrm{SU}(2)$ chiral symmetry and $N_{f}=2$ flavors of fermion. We show that fits to the equation of state are sensitive to the shape and extent of the assumed scaling region; the best fits favour a triviality scenario predicted by the large- $N_{f}$ approximation, in which the scalar degrees of freedom are fermion - anti-fermion composites with wavefunction renormalisation constant vanishing logarithmically in the continuum limit.

In this talk I report on work done with John Kogut; a more detailed presentation has appeared [1]. We have studied the critical behaviour of the four-dimensional Nambu - Jona-Lasinio (NJL) model, whose continuum Lagrangian density is given by

$$
\begin{aligned}
\mathcal{L} & =\bar{\psi}_{i}(\not \partial+m) \psi_{i}-\frac{g^{2}}{2 N_{f}}\left[\left(\bar{\psi}_{i} \psi_{i}\right)^{2}-\left(\bar{\psi}_{i} \gamma_{5} \vec{\tau} \psi_{i}\right)^{2}\right] \\
& =\bar{\psi}_{i}\left(\not \partial+m+\sigma+i \vec{\pi} \cdot \vec{\tau} \gamma_{5}\right) \psi_{i}+\frac{N_{f}}{g^{2}} \operatorname{tr} \Phi^{\dagger} \Phi, \quad(1)
\end{aligned}
$$

where $i$ runs over $N_{f}$ distinct fermion flavors, and in the second line we introduce an auxiliary scalar field $\Phi=\sigma+i \vec{\pi} \cdot \vec{\tau}$ proportional to an $\mathrm{SU}(2)$ matrix. The Lagrangian (1) has an $\mathrm{SU}(2)_{L} \otimes \mathrm{SU}(2)_{R}$ chiral symmetry $\psi_{L} \mapsto U \psi_{L}$, $\psi_{R} \mapsto V \psi_{R}, \Phi \mapsto V \Phi U^{\dagger}$, which is broken explicity by the bare fermion mass, and spontaneously by the development of a condensate $\Sigma \equiv\langle\sigma\rangle \neq 0$.

The NJL model has been proposed to describe both strong [2] and weak [3] interaction physics; in the latter case the Higgs is a composite $f \bar{f}$ state. Both the NJL model and the conventional model of the Higgs sector, namely the $\mathrm{O}(4)$ linear sigma model, are believed trivial in the sense that neither has an interacting continuum limit. It has been shown that the lattice-regularised models have equivalent predictive power for electroweak phenomenology 惯.

In this study we aim to characterise the triviality of the NJL model by fitting numerical data

\footnotetext{
*PPARC Advanced Fellow
}

for $\Sigma$ to the model equation of state (EOS)

$$
m=A\left(\frac{1}{g^{2}}-\frac{1}{g_{c}^{2}}\right) \frac{\Sigma}{|\ln \Sigma|^{q_{1}}}+B \frac{\Sigma^{3}}{|\ln \Sigma|^{q_{2}}} .
$$

The logarithms are corrections to the EOS predicted by mean field theory. Their presence signals triviality: the interacting theory becomes illdefined in the continuum limit $\Sigma \rightarrow 0$ (in lattice units). Eq. (2) has wide applicability; for the $\mathrm{O}(N)$ sigma model the exponents $q_{1,2}$ can be calculated using RG-improved perturbation theory [5]. For the NJL model, they can be estimated to leading order in the $1 / N_{f}$ expansion [6], and are qualitatively distinct (see table). We wish to understand whether this difference is real or merely an artifact of the approximation.

We have simulated a discretised version of (11) on a $16^{4}$ lattice for $N_{f}=2$ [运. Since we use staggered fermions, this necessitates a fractional number of lattice flavors, and hence the use of a hybrid molecular dynamics algorithm. We have accumulated a high statistics dataset with bare mass values in the range $m=0.05, \ldots, 0.0025$. We also performed simulations directly in the chiral limit $m=0$. In this case $\Sigma$ vanishes in a finite volume, so instead we measured the mean field $|\Phi|$ defined via

$|\Phi|=\left\langle\sqrt{\frac{1}{2} \operatorname{tr} \bar{\Phi}^{\dagger} \bar{\Phi}}\right\rangle \quad ; \quad \bar{\Phi}=\frac{1}{V} \sum_{x} \Phi(x)$.

In order to find a satisfactory fit to (2) it is necessary to make some assumptions about the 
Table 1

Values for the correction exponents

\begin{tabular}{lrrrrr}
\hline & Analytical predictions & & & Numerical fits & \\
\cline { 2 - 3 } \cline { 5 - 6 } & $\mathrm{O}(4)$ sigma model & large- $N_{f}$ NJL & & ferromagnetic & composite \\
\hline$q_{1}$ & 0.5 & 0.0 & & $0.786(19)$ & $0.016(11)$ \\
$q_{2}$ & 1.0 & -1.0 & & $0.372(17)$ & $-0.553(18)$ \\
$\chi^{2} /$ dof & & & & 2.5 & 4.6 \\
\hline
\end{tabular}

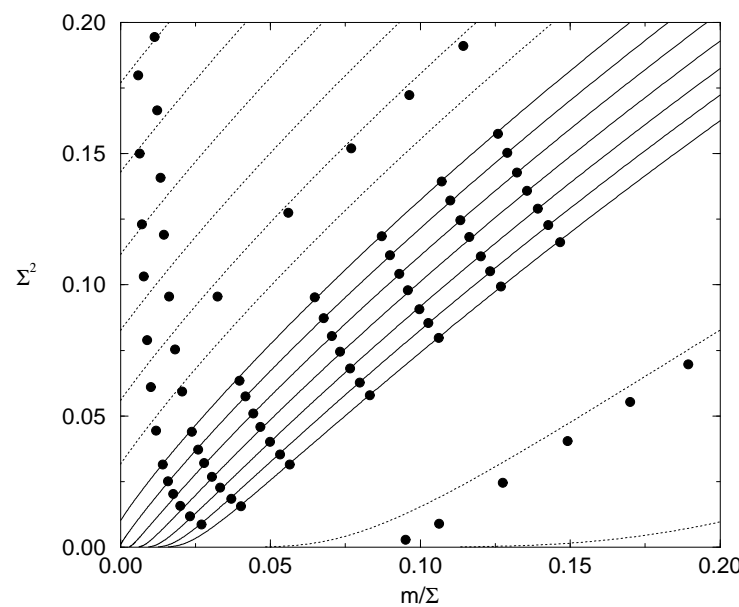

Figure 1. Fisher plot of the ferromagnetic fit.

size and shape of the scaling window about the critical point, and hence which data to include. We have found that our results for $q_{i}$ depend very sensitively on the window chosen. For instance, if we include all mass values, but exclude couplings outside the range $[0.52,0.55]$ then we obtain values, shown in the table, which qualitatively resemble the analytic prediction for the $\mathrm{O}(4)$ sigma model: we label this fit "ferromagnetic". If, on the other hand, we include all coupling values but exclude masses greater than 0.01 , then the fit changes, the value of $q_{1}$ becoming almost zero, and $q_{2}$ becoming negative. We refer to this fit as "composite" because it agrees with the $1 / N_{f}$ treatment of the NJL model. The results of the fits are displayed in Figs. 1, 2 in the form of a

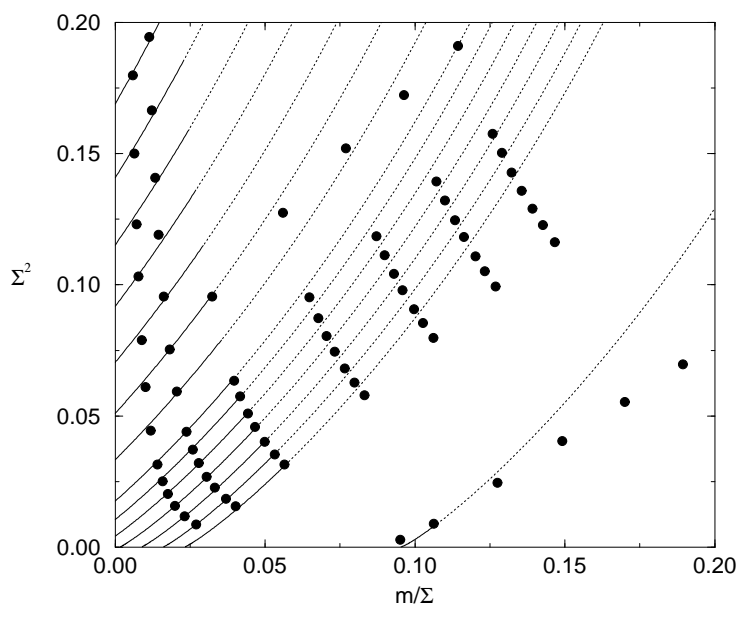

Figure 2. Fisher plot of the composite fit.

Fisher plot of $\Sigma^{2}$ vs. $m / \Sigma$. This would yield straight lines of constant coupling as $m \rightarrow 0$ for a mean field EOS.

It would appear that logarithmic corrections are hard to pin down from EOS fits alone. Both fits have sufficiently large $\chi^{2}$ to be questionable. Fortunately there is an an alternative probe, involving data for $|\Phi|$ taken at $m=0$. On a finite system we expect $|\Phi|$ to exceed $\Sigma_{0}$, the true order parameter obtained by extrapolating the EOS to the chiral limit, because $|\Phi|$ also receives contributions from fluctuations of Goldstone modes, which average to zero only in the thermodynamic limit. The difference $\Delta=|\Phi|-\Sigma_{0}$ can be calculated using chiral perturbation theory to be of 


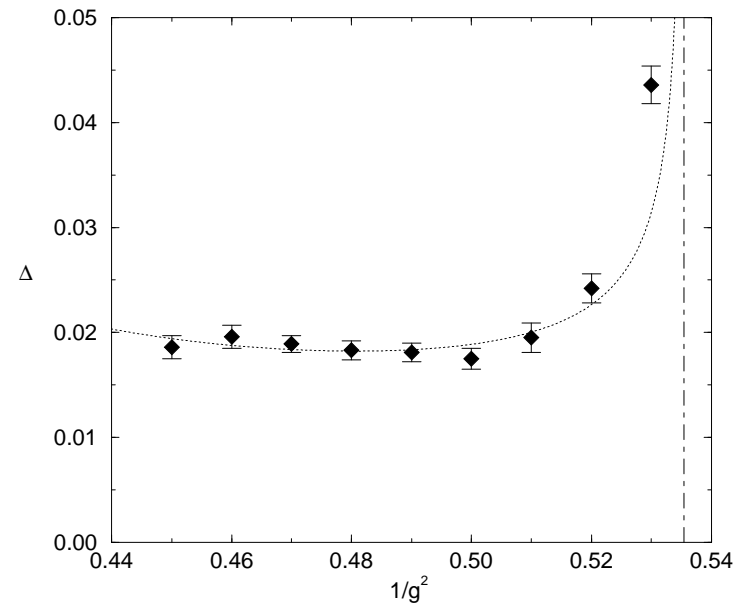

Figure $3 . \Delta$ vs. $1 / g^{2}$ : the vertical line shows the fit for $1 / g_{c}^{2}$

the form [7]

$\Delta \propto \frac{Z}{\Sigma_{0} L^{2}}$,

where $Z$ is the wavefunction renormalisation constant, defined as the coefficient of $1 / p^{2}$ in the pion propagator in the IR limit. The values of $\Delta$ obtained using the chiral extrapolation of the composite EOS fit obtained by setting $q_{1}=0$, $q_{2}=-1$ [1] are shown as a function of $1 / g^{2}$ in Fig. 自 $(\Delta$ extracted using the ferromagnetic EOS changes sign over the range and makes no physical sense).

At first sight the data look incompatible with (位) since $\Delta$ is approximately constant for $1 / g^{2} \in$ $[0.45,0.52]$, while $\Sigma_{0}$ falls by a factor of 3 in the same range. However, we must take into account the dependence of $Z$ on $\Sigma_{0}$. For models such as the sigma model in which the Higgs is elementary, $Z$ is perturbatively close to 1 approaching the continuum limit, and hence $\Delta \propto 1 / \Sigma_{0}$. For a composite Higgs, however, we expect $Z$ to vanish logarithmically in the same limit 6] [8], and hence $\Delta \propto 1 / \Sigma_{0}\left|\ln \Sigma_{0}\right|$. The latter form yields a reasonable fit as shown in Fig. B.
To conclude, this independent study of the finite volume correction seems to prefer the composite EOS predicted in the large- $N_{f}$ approach. It remains an interesting open question whether this result is generic to four-dimensional models in which scalars are composite, or whether other universality classes exist defined by alternative lattice formulations [9].

\section{REFERENCES}

1. S.J. Hands and J.B. Kogut, hep-lat/9705015 (1997).

2. Y. Nambu and G. Jona-Lasinio, Phys. Rev. 122 (1961) 345.

3. Y. Nambu, in New Trends in Physics, eds. Z. Adjuk, S. Pokorski and A. Trautman (World Scientific, Singapore, 1989):

V.A. Miransky, M. Tanabashi and K. Yamawaki, Mod. Phys. Lett. 62 (1989) 1433;

W. Bardeen, C. Hill and M. Lindner, Phys. Rev. D41 (1990) 1647.

4. A. Hasenfratz et al, Nucl. Phys. B365 (1991) 79.

5. E. Brézin, J.C. Le Guillou and J. Zinn-Justin, in Phase Transitions and Critical Phenomena, vol. 6, eds. C. Domb and M. Green (Academic Press, London, 1976).

6. A. Kocić and J.B. Kogut, Nucl. Phys. B422 (1994) 593..

7. M. Göckeler and H. Leutwyler, Nucl. Phys. B350 (1991) 228;

M. Göckeler et al, Nucl. Phys. B404 (1993) 517.

8. T. Eguchi, Phys. Rev. D17 (1978) 611;

K.I. Shizuya, Phys. Rev. D21 (1980) 2327.

9. A. Ali-Khan et al, Phys. Rev. D51 (1995) 3751. 